\title{
Glyphosate nontoxicity: the genesis of a scientific fact
}

\author{
Marek Cuhra ${ }^{1,2, *}$ \\ ${ }^{1}$ GenØk-Centre for Biosafety, The Science Park, P.O.Box 6418, 9294 Tromsø, Norway \\ ${ }^{2}$ Faculty of Health Sciences UiT, Arctic University of Norway, Tromsø, Norway
}

\begin{abstract}
Repetition of a 1978 experiment on the toxicity of glyphosate chemicals in water-flea Daphnia magna showed surprising results. In the 31 years which had passed between the two series of experiments, the toxicity of glyphosate had apparently become 300 times stronger! Further investigation into this enigmatic paradox discloses unfortunate aspects of laboratory researcher cultures as well as fundamental challenges in current regulatory approval of chemicals and the epistemology of risk-assessment.
\end{abstract}

Keywords: flexibility of scientific facts, glyphosate toxicity, scientific fraud

\section{INTRODUCTION}

When biochemical inventions or new chemical compounds with a potentially large impact on health or the environment are to be assessed for approval and release onto the market, it is of the utmost importance that possible effects are investigated and anticipated. In our world, many such inventions and novel products emerge from commercial initiatives. Often, even discoveries made in the publicly funded laboratories of universities and state research institutions are put on the market by private commercial interests [1].

The primary assessment of any novel chemical will evaluate whether the substance actually works as intended and whether it has any unintended effects, according to defined tests and anticipated categories of results. In order to safeguard this precautionary approach, society has developed norms for testing and evaluation within regulatory, institutional and administrative framework of national and international bodies. Authorities such as the World Health Organization (WHO), the Organization for Economic Coöperation and Development (OECD), the European Food Safety Authority (EFSA), the United States Food and Drug Administration (US FDA) and Environmental Protection Agency (US EPA) should be mentioned as important sources of scientific advice for policymakers; they collect relevant evidence and assess the possible negative effects of products in order to establish guidelines for safe use. The resulting regulatory framework is seen as governing the actions and impact of the chemical industry, the biomedical industry and the food-producing industry.

Policies regarding individual products, drugs and chemicals are thus regulated on the basis of findings from scientific research. The complex ways in which individual tests and research results are produced, collected and assembled into the foundations of regulatory documents, policy guidelines and norms of decision-making makes it worthwhile to take a closer look at the underlying mechanisms involved.

It is not uncommon that research conducted by individual scientists or communities of scientists show contradictory results on the same subject, possibly due to using different methods or interpreting data differently. Numerical data are often challenging to interpret, with various possible statistical analyses and measures of significance broadening the standard methods of assessment. The diversity of views can thus stem from differences in methodology and individual interpretation of evidence. Less evident, and seemingly objectively irrelevant, factors can also influence the direction of scientific conclusions, such as the economic implications of certain research findings, prevailing science-based policies or simple personal conflicts and rivalry. This all enhances the importance of good clear research protocols and defined methods for data collection and interpretation, thus making research findings reproducible.

Finally, we must acknowledge that scientific methods, normality of research procedures and the daily routines in the laboratory are all influenced by what has been described as "the professional culture of scientists" [2]. In this context it is important to realise that our society expects the industry itself to provide the main evidence for the safety assessment of their chemicals. Thus, the responsibility for carrying out testing and interpreting the data has been delegated to the industry that aspires to place commercial products onto the market. Some independent researchers have voiced clear reservations against such autonomy, propagating the view that trust is good but control is better [3].

\footnotetext{
*E-mail:marek.cuhra@gmail.com; tel: +4799585427; fax: +4777646100
} 


\section{A CASE STUDY}

The following case concerns the risk assessment of a chemical compound that has gradually become the most important agrochemical globally. The case is based on data from hitherto confidential product information in the archives of the US EPA, extracted from 21 individual safety studies and related documentation, which was obtained through a recent Freedom of Information Act request [4].

In 1978 a group of scientists at the Analytical BioChemistry (ABC) laboratories in Columbia, Missouri were commissioned by the chemical company Monsanto to test a new, promising chemical. The substance was glyphosate and Monsanto had high expectations for it; it was found to have very promising effects on practically all kinds of weeds in agriculture. Glyphosate was initially proposed as a selective herbicide, only active against growing plants and thus ideal for field clearance prior to sowing.

In order to assess possible undesirable effects of glyphosate, the scientists at $\mathrm{ABC}$ carried out tests using nontarget organisms and human models. Several of those laboratory tests were designed to assess glyphosate toxicity towards aquatic organisms such as fish and invertebrates. Researchers William A. McAllister and Alan D. Forbis of the ABC research team assessed the immediate and long-term toxicity of glyphosate in aquatic invertebrates by testing glyphosate in the common water flea Daphnia magna, a universal indicator organism used in hundreds of research laboratories all over the world. In 48-hour short-term studies (mortality testing) the scientists saw no effect for concentrations of up to $560 \mathrm{mg} / \mathrm{L}$ and the resulting $\mathrm{EC}_{50}$ value (the concentration at which $50 \%$ of Daphnia die in 48 hours) was determined as $780 \mathrm{mg} / \mathrm{L}$. Using the toxicity categories valid at the time, the researchers concluded that the results "would place technical glyphosate into the category of practically nontoxic" [5]. Another ABC test, of the isopropylamine (IPA) salt of glyphosate in D. magna, was reported a few years later and, apparently, showed even lower toxicity, yielding an $\mathrm{EC}_{50}$ value of $930 \mathrm{mg} / \mathrm{L}$ [6]. Furthermore, the research records show that concentrations of up to $50 \mathrm{mg} / \mathrm{L}$ of glyphosate in the test water had no negative effect in long-term (21 days) experiments. The data produced by $\mathrm{ABC}$ were submitted to the US EPA and approved a few years later. The results were actively used by both Monsanto and regulatory authorities as evidence for the nontoxicity of glyphosate herbicides for aquatic invertebrates. Along with the results from over 250 other experiments [7], these results from the $\mathrm{ABC}$ laboratories were later compiled into an extensive ecotoxicological risk assessment for glyphosate and Roundup (a commercial herbicide formulation in which glyphosate is the main active ingredient) published in a reputable academic journal [8]. The nontoxicity of glyphosate had thus become a scientific fact. The problem was, however, that the mentioned data produced by the $\mathrm{ABC}$ laboratories could not be subsequently reproduced by other researchers repeating the experiments. The differences were not negligible, since the subsequent research [9-12, 33] showed these chemicals to be up to 300 times more toxic than reported by $\mathrm{ABC}$.

\section{WHAT IS A SCIENTIFIC FACT?}

In the definition given by Polish scientist Ludwik Fleck, ${ }^{1}$ "A fact is supposed to be distinguished from transient theories as something definite, permanent, and independent of any subjective interpretation by the scientist" [13]. Another simple definition can be extrapolated from the metaphors "The Cat is on the Mat" and "Brains in a Vat" presented by Hilary Putnam in his analysis of observable fact [14]. Either the cat is on the mat, or the cat is not on the mat. The research done by McAllister and Forbis at ABC was of exactly this simple nature; at a given concentration of the specific chemical, the individual daphnid was either alive or dead. The fact in this experimental setup is derived from simple counting of daphnia alive versus daphnia dead in progressively increasing concentrations of the chemical, yielding binary data which are routinely processed in probit regression analysis to determine the exact $\mathrm{EC}_{50}$. In this scientific procedure, there is very limited room for subjective interpretation that could potentially influence the validity of the scientific fact. This type of scientific research can be seen as observation in its purest form; the daphnid is either dead or alive. Still, we get this surprising report from relatively high concentrations of the chemical; the daphnids were reported as seen to be alive by McAllister and Forbis, but according to subsequent findings by other researchers, they would be expected to be dead. Hence, McAllister and Forbis saw the cat on the mat, in a situation where no other scientist has apparently been able to see it.

How can this be possible? Could it be that McAllister and Forbis imagined that they saw the cat?

\footnotetext{
${ }^{1}$ In his pre-World War 2 book "Entstehung und Entwicklung einer wissenschaftlichen Tatsache: Einführung in die Lehre vom Denkstil und Denkkollektiv" [13], which was translated into English and published in 1979 as "Genesis and Development of a Scientific Fact" (a title unfortunately impoverished by translation).
} 
This is highly unlikely, as their research protocol clearly states the proportions of daphnids that were alive, as well as those that were dead. At the no-effect concentration of $560 \mathrm{mg} / \mathrm{L}, \mathrm{McA}$ Allister and Forbis reported they saw all 30 daphnids alive 48 hours into the experiment. Or, maybe what McAllister and Forbis were actually reporting was not "the cat on the mat", but instead "the cat on the carpet". And what might the difference be? Well, there are several chemicals named glyphosate but, in herbicide formulations, only various glyphosate salts, such as the commonly used isopropylamine derivative, are of relevance. This type of glyphosate is water-soluble and constitutes the active ingredient in herbicide formulations. Thus, glyphosate IPA salt is often labelled simply glyphosate. But primary forms of glyphosate from earlier stages in the production process are also commonly known as glyphosate. Such primary forms have very low solubility in water and are thus useless as herbicides intended to be diluted with water prior to use, as glyphosate herbicides are. But, if this was the chemical that Monsanto supplied to McAllister and Forbis, and if this was the chemical that McAllister and Forbis subsequently tested, then both parties may have engaged in a type of scientific fraud. It is not a scientifically accepted test of toxicity to test water-insoluble chemicals in a D. magna toxicity study. It simply would not make sense, the chemical would be at the bottom of the container in its crystalline form and the test animals would not be affected by it. When reviewing the analytical report prepared by McAllister and Forbis, it is interesting to note one of several hand-written additions to the typed report. It is in the section describing the chemical tested, where the typed wording "glyphosate" has been supplemented by the US EPA code \#103601. This chemical code identifies N-(phosphonomethyl)glycine monoisopropylamine salt, or simply the IPA salt of glyphosate, which, as mentioned, is the most relevant chemical form of glyphosate in herbicide formulations [15]. But, why was the hand-written chemical identity specifying the IPA salt added to the report? Was it to specify, or was it actually to falsify? And who did it, and when?

\section{HOW DO WE OBTAIN FACTS FROM RESEARCH ENDPOINTS?}

The mentioned standardized $\mathrm{EC}_{50} D$. magna test of acute toxicity is an example of an exact test, with clearly defined research endpoints that can be used in assessing immediate unintended effects of (in this example) a new agricultural pesticide on the biodiversity of lakes and ponds, defined as "nontarget aquatic invertebrate organisms". Several detailed operating procedures are defined for this specific test, among which the best known and most widely used are industry tests such as the ASTM D. magna acute toxicity test, the ISO-standard D. magna acute toxicity test, international organization tests such as the OECD 202 D. magna acute toxicity test and national tests such as the US EPA OPPTS 850.1010 Daphnia acute toxicity test. In addition to those, there are numerous tests applied at institutional level, such as the guidelines we use in our D. magna testing laboratory at the University of Troms $\varnothing$ and similar guidelines used by our colleagues here in Norway and abroad. To fully understand the limits of testing, we must bear in mind that these mentioned guidelines are for only one single test, namely the assessment of acute toxicity in aquatic invertebrates, represented by the test species Daphnia magna (sometimes other similar species of daphnids are used, but the main principles are the same). Such a test only investigates one singular component of the tested substance, the endpoint being the immediate (acute) toxicity to aquatic invertebrate organisms, measured as mortality (or paralysis or immobility) in Daphnia. In addition to such daphnia tests, tests using algae, fish, insect larvae and other categories of organisms from freshwater environments are carried out. For some categories of organisms, such as fish, it is recommended to test several different species. Typically the $\mathrm{EC}_{50}$ mortality tests are done for an exact short interval, most often 24, 48 or 96 hours, according to test protocol and species.

Another category of test guidelines is used to assess possible long-term toxic effects (chronic toxicity tests) in aquatic invertebrates. Among these, the US EPA test for chronic toxicity in freshwater organisms [16] and the OECD 211 D. magna reproduction test [17] are recognized as international standards. Such lifelong tests are well suited to reveal possible less-evident toxic effects on growth, long-term survival and reproduction.

The scope of all these tests is to garner evidence for possible effects on aquatic invertebrates investigated through the indicator species Daphnia and, thus, the conclusions are limited to that. For a holistic approach to possible environmental effects of a specific compound, it is necessary to organize testing with many additional species and categories of organisms, such as plants, microörganisms, invertebrates, birds, mammals and humans. Tests restricted to in vitro laboratory environments must be supplemented by assessment of ecosystem effects through field studies. Such holistic approaches are increasingly relevant for chemicals such as new pesticides (comprising herbicides, insecticides, fungicides etc.) and other potentially harmful compounds intended for deliberate release into the environment (other régimes apply for the testing of pharmaceutical chemicals, with a direct focus on human health). 
Mostly, such tests are organized and financed by the industrial interests behind the product. Thereby the industry takes not only financial and practical responsibility for the relevant tests, but also accepts a substantial ethical challenge; conducting testing in the correct manner and ensuring the transparency and reproducibility of such testing. The results obtained are presented to the relevant national or international regulatory authorities when applying for authorization to release the product into the consumer market or the environment. In order to comprehend and truly understand the scientific results from such specific individual tests in context, it is common to work up some sort of synthesis. One such synthesis is the already mentioned review [8] of glyphosate and Roundup ecotoxicity. The questionable data from McAllister and Forbis penetrated not only into that review, but can be found subsequently in innumerable pieces of documentation, ranging from simple data sheets to international policy statements on glyphosate herbicides. In some of these documents the original source of the data has fallen into oblivion, such as in a 2010 review on glyphosate by Syracuse Environmental Research Associates Inc. (SERA), which reports the $780 \mathrm{mg} / \mathrm{L} \mathrm{EC}_{50}$ value as originating from a US EPA $1993 \mathrm{RED}^{2}$ on glyphosate $[18,32]$. The data from $\mathrm{ABC}$ on glyphosate toxicity in Daphnia travelled overseas, as they are now incorporated into European Union legislation in the European Commission working document on glyphosate [19]. In this important document, it is the $\mathrm{EC}_{50}$ value of $930 \mathrm{mg} / \mathrm{L}$ from the ABC 1981 study conducted by Forbis and Boudreau that is used, presumably because this study specifies more clearly that the tested type of glyphosate is the IPA salt, or possibly since this specific study shows even less toxicity than the 1978 study by McAllister and Forbis. In 1994, the WHO published its environmental health criteria (EHC) report on glyphosate [20], as an extensive review based on a substantial part of the evidence available at the time. The report has a detailed chapter on ecotoxicological effects in aquatic invertebrates, but it is rather contradictory, since although it presents the latest data on Daphnia sensitivity to glyphosate and glyphosate herbicides, it is also infected by the results originating from $\mathrm{ABC}$, hence the "synthesis" is quite confusing. In addition to the unpublished $\mathrm{ABC}$ studies mentioned before, WHO also quotes a newer $\mathrm{ABC}$ study on chronic toxicity of glyphosate in Daphnia from 1989, where ABC researchers reportedly found NOEC levels of $100 \mathrm{mg} / \mathrm{L}{ }^{3}$ Based on the trade record of the $\mathrm{ABC}$ laboratories, again, this is a test result that we must interpret as highly questionable evidence of safety. Paradoxically, the WHO report presents a table of test conditions, specifying factors such as the $\mathrm{pH}$ and hardness of the test water in the 1978 study by McAllister and Forbis, but this is some sort of mistake, since the original report does not specify these parameters. The latest known revision of glyphosate ecotoxicity is the above-mentioned 2010 evaluation of glyphosate by SERA for the USDA Forest Service [18]. In this cornerstone review on glyphosate, the following quote can be found: "McAllister and Forbes 1978b, MRID 00108172. This study is cited on several MSDSs", indicating that the ABC results have been used as evidence of safety in several material safety data sheets for different glyphosate herbicides. A brief search discloses at least six such data sheets for different commercial glyphosate herbicides in present use in the USA, which have not been actually tested for aquatic invertebrate toxicity; the data sheets merely quote the $780 \mathrm{mg} / \mathrm{L}$ value from McAllister and Forbis. There is reason to believe that these six formulations contain the IPA salt of glyphosate, as this is the main type of glyphosate used in herbicide formulations.

The SERA report is a somewhat overwhelming and confusing document, where terms are mixed and not all conclusions are justified. Nevertheless, from such an enormous review some interesting information can be extracted if one has the necessary patience. ${ }^{4}$ Also, even though questionable, the reference material informs us on other aspects of this case: On page 175 we find the following reference: "MRID: 101533 Danhaus, R.; Lenox, E.; Dubelman, S.; et al. (1982). Dissipation of Alachlor in Field Soils following Preemergent Applications of Lasso ME Alone or in Tank Mix Combinations with Roundup, Atrazine, Dyanap, Metribuzin or Cyanazine: MSL-2109 (unpublished study received May 10, 1982 under 524-344; prepared in cooperation with ABC Laboratories, Inc. and Craven Laboratories, Inc., submitted by Monsanto Co., Washington, DC; CDL:070841-D)." As we shall see in the following, this information is

\footnotetext{
${ }^{2}$ The US EPA Reregistration Eligibility Decision (RED) on glyphosate [32] is the main regulatory document on glyphosate in the US public administration.

3 Title: 21-day prolonged static renewal toxicity of glyphosate technical to Daphnia magna. Unpublished study.

${ }^{4}$ SERA 2010 supplement 1 is itself quite a bulky document of 464 pages with a collection of 5652 references to "Registered submitted studies on glyphosate" as of March 2010. It must be mentioned that although 20 of these SERA references describe ecotoxicological effects of glyphosate in Daphnia, only 3 references are to peer-reviewed publications that we have been able to access in our work on the subject. The remaining 17 references are to unpublished studies from industry.
} 
important, as it indicates some degree of formalized coöperation between the two mentioned laboratories.

\section{AHISTORY OF SYSTEMATIC FRAUD}

Back in the late 1970s several commercial laboratories working for Monsanto were involved in assessing the toxicity of the new flagship herbicide. One of these service providers was Industrial Bio-Test Laboratories (IBT), which at the time was working for several manufacturers of pesticides. But then, unexpectedly, the IBT laboratories were visited by inspectors from the US FDA and exposed as having systematically produced fraudulent data [21]. A few years later Craven Labs, another laboratory working for Monsanto, was exposed by a "pesticide industry task force", and the US EPA, as having committed scientific fraud [22,23]. The president of IBT was sentenced to four years in prison, but allegedly never served a day of his sentence, because "his heart was bad" [24]. As late as 2001 the US EPA conducted a campaign directed towards "the environmental analytical laboratory community" [25]. Following a series of scandals in the years 1976, 1990 and 1997-2000, which exposed widespread systematic fraud and misconduct in analytical laboratories working for industry clients, the US FDA led several such campaigns to reduce malpractice. This also led to the establishment of laboratory standards, defining first routines of Good Laboratory Practice in 1979 (following the IBT scandal). Later, a task force of inspectors was established, including a hotline for whistleblowers and new rounds of information targeted at the laboratory industry $[22,23]$. In a 2002 report update from the US EPA Laboratory Fraud Work Group, the EPA states; "the ramifications stemming from a laboratory's falsifications spread far beyond the specific tampered results; once the laboratory's integrity is compromised, all the data generated by that laboratory is questionable" [26].

Although two of the laboratories involved in quality assurance and testing of Monsanto products were found guilty of falsifying scientific data, this does not necessarily imply that the above-mentioned data on glyphosate toxicity in Daphnia produced by the ABC laboratories is less credible. The mentioned documentation from the SERA supplements indicates commercial coöperation between the Craven and $\mathrm{ABC}$ laboratories at the time of glyphosate testing and before Craven was found guilty of organized scientific fraud, but so far there is no indication that there has been any systematic irregularity in the work that $\mathrm{ABC}$ produced for Monsanto. However, when we look closely at the test protocols submitted by Mcallister and Forbis, several interesting questions arise.
First of all, the raw data for probit analysis look a bit too perfect. From my own experience, so far having conducted approximately 20 separate acute toxicity tests in Daphnia magna [12], I would expect to see the data express less geometric symmetry in the two lower effect concentrations. But that is by no means conclusive evidence of fraud. However, in their probit analysis, McAllister and Forbis use very closely spaced concentrations $(560,650,750$ and $870 \mathrm{mg} / \mathrm{L})$ and produce an $\mathrm{EC}_{50}$ value of $759.7 \mathrm{mg} / \mathrm{L}$, with an impressively sharp $95 \%$ confidence interval of $740.8-779.9 \mathrm{mg} / \mathrm{L}$. We recalculated the $\mathrm{EC}_{50}$ value from the data provided by McAllister and Forbis and found their computations to be correct. But it is not acceptable scientific practice to present the highend limit of the confidence interval as the $\mathrm{EC}_{50}$ value. The calculated $\mathrm{EC}_{50}$ value of $759.7(760) \mathrm{mg} / \mathrm{L}$ should have been used, not the $780 \mathrm{mg} / \mathrm{L}$ value obtained from the top of the confidence interval. This may seem like a small detail, but it is a clear indication of unsound scientific practice. The review of these reports of research performed at $\mathrm{ABC}$ strongly indicates that unsuitable methodologies have been employed, evinced as flaws in the experimental setup, misinterpretation of the data and miscalculation of endpoints. Furthermore, the regulatory importance of these documents has been exaggerated and scientific conclusions have been changed in subsequent revisions. Also, the documentation indicates that US EPA staff assisted in such manipulation of conclusions.

\section{HOW TOXIC IS GLYPHOSATE TO AQUATIC ORGANISMS IN REALITY?}

Numerous studies show that the true acute toxicity of glyphosate and glyphosate herbicides for D. magna, measured as 48 hours $\mathrm{EC}_{50}$ or $\mathrm{LC}_{50}$ values, is somewhere in the range $2-149 \mathrm{mg} / \mathrm{L}$ depending on herbicide type and test conditions [10,12, 27, 28]. Although this shows glyphosate to be much more toxic than the $780-930 \mathrm{mg} / \mathrm{L}$ values reported by $\mathrm{ABC}$, this chemical does actually still not qualify for a very high degree of toxicity for aquatic invertebrates, and we could use the term "moderately toxic" (in the US EPA 1985 classification) and simply "toxic" (in the European Council 1993 classification). New ways of assessing the toxicity of pesticides are emerging. In 2001 an international workshop on probabilistic methods for risk assessment of pesticides was held in the Netherlands. The 103 participants included international experts in toxicology and probabilistic methods, potential users from government and industry in EU member states and the European Free Trade Association (EFTA) countries, and representatives of the European Commission, the Pesticides Action Network and the American Bird Conservancy. The 
objective of the workshop was to try and work up new ways of thinking, moving from causality to holistic methods: "Broadly speaking, the term probabilistic refers to risk assessments that attempt to quantify variability and/or uncertainty in factors that influence risk, and express risk in terms of the probability and magnitude of adverse effects. One specific area where probabilistic methods might potentially be used is in assessing the impact of plant protection products (pesticides) on the environment. Directive 91/414/EEC requires EU member states to analyze these risks before authorizing pesticides for sale. The methods currently used for these assessments are predominantly deterministic rather than probabilistic; they use fixed values for exposure, toxicity and risk, and attempt to allow for variability and uncertainty by using worst-case assumptions and safety factors. A probabilistic approach would allow for variation and uncertainty by using distributions, instead of fixed values, for exposure, toxicity and risk" [29].

The workshop had a special focus on pesticide toxicity in aquatic organisms and the working group produced a comprehensive list of possible advantages such methods might bring. The European Commission guidance document on aquatic ecotoxicology is open to a new approach to the subject. The document lines up methods for testing in species of Daphnia, and recommends that "uncertainty factors of 100 and 10 are applied to acute and chronic endpoints respectively to account for potential inter-species differences in invertebrate sensitivity and other sources of uncertainty" [19, (ch. 2)]. The document also gives important guidelines for pesticide use, when runoff to an aquatic environment can be expected: "Where there is a possibility of aquatic organisms being exposed, no authorisation should be granted if the toxicity/exposure ratio for fish and Daphnia is less than 100 for acute exposure and less than 10 for long-term exposure" [19, (ch. 4)]. With this type of precautionary approach and using the correct data for glyphosate toxicity in Daphnia, some of the present use of glyphosate herbicides may be questioned.

\section{THE ELASTICITY OF FACTS}

An important issue here relates to the dynamics of scientific evidence from tests. In science, the framework of peer-review is normally used to ensure that only research results of sufficient quality get to be published. Once a result is published, it is seen as part of the universal body of scientific evidence. Other researchers will relate to it, refer to it and compare it to their own findings and the findings of others. Returning to the earlier discussion of the epistemology of facts, "To say that something is a scientific fact is to bring in epistemology and show awareness of the epistemic brittleness of common sense. Advertisers often do it. Many products are sold (sometimes correctly and sometimes wrongly) in the name of science. As consumers, we are often told that products have been scientifically tested, and that the promised effects have been scientifically proven to be there" [30]. This leads directly into another interesting situation to consider about Roundup. In 1996 the Attorney General of the State of New York led a legal case against the agrochemical company Monsanto. The case was spectacular and has been described as legal action to stop false advertising of Roundup herbicide, but it must also be seen as a legal case on the interpretation of scientific data. The case was prepared by Michael H. Surgan, who at the time was the chief scientist in the New York State Attorney General's Environmental Protection Bureau. A brief search brings up several peer-reviewed papers by this chief scientist, hence arguably the Attorney General had the necessary professional competence in his team at hand, prepared to venture into scientific argumentation. The result of the case was a long list of general restrictions on Roundup advertising practice and also specific rulings in scientific questions. One such restriction specified that future toxicity characterization of the Roundup herbicide was not to be taken out of context in future presentation or public debate by the manufacturer; thus, claims of the herbicide being "practically nontoxic" or "slightly toxic" were to be substantiated by additional information on the test method, the toxic effect evaluated (endpoints), the exposure routes tested and the tested species. In addition, the Attorney General stated that "to the extent that any representations are based on data for individual components (e.g., the active ingredient), rather than for mixtures (formulated product) as applied, such representations must be clear as to whether the claim is being made for the active ingredient or for the product" [31]. Thus, the Attorney General entered the scientific debate on whether pesticides should be assessed on the basis of their active ingredients alone, or whether the so called "inert ingredients" and various chemical agents present in commercial formulations of pesticides should be assessed as having an equal or potentially even higher impact on health and the environment than that of the active ingredient alone.

\section{WHITEWASHING OF DATA}

The most important and damaging effect of the misrepresentation of glyphosate toxicity in Daphnia caused by the data presented by McAllister and Forbis has been the subsequent incorporation of these data into the framework of regulation: Not only as universal 
reference for the alleged low toxicity of glyphosate herbicides in general but, more importantly, by seeping into, and being uncritically incorporated into, the body of scientific evidence on glyphosate and Roundup toxicity. We see that by presenting the data from the $\mathrm{ABC}$ testing, authors from the independent scientific community have conferred onto these misleading data the legitimacy of real facts, incorporating them into comprehensive reviews and publishing these reviews in esteemed peerreviewed scientific journals. Following this, regulators such as the US EPA and EFSA, as well as consultants working in policy document preparation, have absorbed and presented these misleading data as if they were true. Through a process of innumerable reduplication and cross-referencing, the data from a small and highly questionable experiment in a private laboratory working for Monsanto in 1978 have become scientific fact in the present time. This is even more paradoxical when we see that still more tests have been done on the toxicity of glyphosate and glyphosate herbicides in recent years. We have collected numerous peer-reviewed investigations into this specific scientific question, by researchers working in various countries [12]. The great majority of this work indicates the same as our own testing of glyphosate and Roundup in Daphnia, namely that the original work by McAllister and Forbis is not representative. Then, why is the work of McAllister and Forbis still being presented as the truth in revision after revision of policy documents on these chemicals? Through our investigation into the subject we have become convinced that it is due to a series of unfortunate circumstances: firstly by the fact that these data penetrated into peer-reviewed publications such as widely used reference works; secondly, because regulators do not have a mechanism of revising such fundamental information other than the process of updating reviews, guidelines and policy documents and, although that process is based on peer review and public hearings, these matters are often so immensely complex that only a few of the scientists involved (even those sitting on the expert panels) have the capacity to submerge themselves into the details; thirdly: companies producing glyphosate herbicides use "interpretations" of the $780 \mathrm{mg} / \mathrm{L}$ and $930 \mathrm{mg} / \mathrm{L} \mathrm{EC}_{50}$ values, often presenting some obscurely derived fraction of these values based on the glyphosate content in their specific product; this unfortunately gives the impression that independent tests have been made and thus contributes further "new evidence". One paradoxical aspect of this whole matter, is the fact that the scientific misconduct perpetrated by researchers at $\mathrm{ABC}$, disclosed and described here, seems to have been unnecessary. Even though our new assessments and reviews [12] have found that the lethal toxicity level for glyphosate is of the order of $5 \mathrm{mg} / \mathrm{L}$ in Daphnia (defined as $\mathrm{EC}_{50}(48)$ values), then that still only indicates moderate toxicity. In any case, none of the credible evidence I have reviewed in my 5-year study of this subject has shown that glyphosate should be termed highly toxic to the Daphnia test species. So, if the true results show moderate toxicity, then why did the researchers at $\mathrm{ABC}$ cheat and falsify the data? Or could it be that they just performed the testing in the "standard way" used at the time? Well, for one thing, nobody at the time could have known that the substance they were testing was to become the world's most important and most widely used agrochemical and, thus, a subject for future scrutiny and repeated testing.

\section{ACKNOWLEDGMENTS}

This work has been funded by Forskningsrådet the Norwegian Research Council, project no. 184107 Miljø-2015. The author wishes to express appreciation for constructive scientific and stylistic contributions from anonymous reviewers and the editorial board, respectively.

\section{REFERENCES}

1. David, P.A., Hall, B.H. and Toole, A.A. Is public R\&D a complement or substitute for private R\&D? A review of the econometric evidence. Research Policy 29 (2000) 497-529.

2. Knorr-Cetina, K. Epistemic Cultures: How the Sciences Make Knowledge. Cambridge, Mass.: Harvard University Press (1999).

3. Tweedale, T. Good laboratory practices and safety assessments: another view. Environ. Health Perspect. 118 (2010) 194-195.

4. FOIA request HQ-FOI-00325-12. United States Environmental Protection Agency, Washington, DC.

5. McAllister, W. and Forbis, A. Acute Toxicity of Technical Glyphosate (AB-78-201) to Daphnia magna. Unpublished study reviewed and approved $8-30-85$ by EEB/HED US EPA(1978).

6. Forbis, A.D. and Boudreau, P. Acute toxicity of MON0139 (Lot LURT 12011)(AB-81-074) to Daphnia magna: Static acute bio-assay report no. 27203. Unpublished study document from US EPA library (1981).

7. Monsanto Backgrounder: Summary of Ecotoxicological Risk Assessment for Roundup ${ }^{\circledR}$ Herbicide (2005) (http:// www.monsanto.com/products/documents/glyphosatebackground-materials/ecotoxicological_risk.pdf).

8. Giesy, J., Dobson, S. and Solomon, K.R. Ecotoxicological risk assessment for Roundup ${ }^{\circledR}$ herbicide. Rev. Environ. Contam. Toxicol. 167 (2000) 35-120.

9. Folmar, L.C., Sanders, H.O. and Julin, A.M. Toxicity of the herbicide glyphosate and several of its formulations to fish and aquatic invertebrates. Arch. Environ. Contam. Toxicol. 8 (1979) 269-278.

10. Melnichuk, S.D., Shcherban, E.P. and Lokhanskaya, V.I. Estimation of toxicity of glyphosate-based herbicide by biotesting method using Cladocera. Hydrobiological J. $\mathbf{4 3}$ (2007) 80-91. 
11. Tsui, M.T.K. and Chu, L.M. Aquatic toxicity of glyphosatebased formulations: comparison between different organisms and the effects of environmental factors. Chemosphere $\mathbf{5 2}$ (2003) 1189-1197.

12. Cuhra, M., Traavik, T. and Bøhn, T. Clone- and agedependent toxicity of a glyphosate commercial formulation and its active ingredient in Daphnia magna. Ecotoxicology 22 (2013) 251-262.

13. Fleck, L. Entstehung und Entwicklung einer wissenschaftlichen Tatsache: Einführung in die Lehre vom Denkstil und Denkkollektiv. Basel: Verlag Benno Schwabe \& Co (1935).

14. Putnam, H. Reason, Truth and History. New York: Cambridge University Press (1981).

15. Chemindustry CAS 103601: Acronyms for glyphosate IPA-salt (2011) (http://www.chemindustry.com/chemicals/ 03561890.html).

16. Short-Term Methods for Estimating the Chronic Toxicity of Effluents and Receiving Waters to Freshwater Organisms. Washington, DC: United States Environmental Protection Agency (2002).

17. OECD-211 Guidelines for Testing of Chemicals: Daphnia magna Reproduction Test. Geneva: OECD (2008).

18. SERA TR-052-22-03b. Glyphosate Human Health and Ecological Risk Assessment. Final report to USDA (Forest Service, Atlanta, Georgia). Manlius, New York: Syracuse Environmental Research Associates, Inc. (2010).

19. EC Working Document: Guidance Document on Aquatic Ecotoxicology in the Context of Directive 91/414/EEC. European Commission Health \& Consumer Protection Directorate General (2002) (http://ec.europa.eu/food/plant/ protection/evaluation/guidance/wrkdoc10_en.pdf).

20. International programme on chemical safety. Environmental Health Criteria 159: Glyphosate. Geneva: World Health Orgnization (1994).

21. Schneider, K. Faking it: The case against industrial bio-test laboratories. The Amicus Journal. Natural Resources Defense Council (NRDC) (1983) (http://planetwaves.net/ contents/faking_it.html, accessed September 2015).

22. Novak, R.A. The long arm of the lab laws. The Chemist $\mathbf{1 0}$ (2001) 45-46.

23. Monsanto Imagine Backgrounder: Testing fraud: IBT and Craven Laboratories (2005) (http://www.monsanto.com/ products/documents/glyphosate-background-materials/ ibt_craven_bkg.pdf).

24. Cummins, J. Re: (SANET-MG) Biotest Lab pesticide fraud of 1970s (2005) (http:/www.ibiblio.org/echolandtech/ SoilWiki/message-archives/JoeCummins/msg00440.html).

25. EPA Open letter to the environmental analytical laboratory community. Washington, DC: US EPA Inspector General's Office, 5 September 2001.

26. Report on the Laboratory Fraud Work Group. US EPA Office of Criminal Enforcement, Forensics, and Training (2002).

27. Dominguez-Cortinas, G., Saavedra, J.M., Santos-Medrano, G.E. and Rico-Martinez, R. Analysis of the toxicity of glyphosate and Faena(r) using the freshwater invertebrates Daphnia magna and Lecane quadridentata. Toxicol. Environ. Chem. 90 (2008) 377-384.

28. Sánchez-Bayo, F. Comparative acute toxicity of organic pollutants and reference values for crustaceans. I. Branchiopoda, Copepoda and Ostracoda. Environ. Pollution 139 (2006) 385-420.

29. Probabilistic Risk Assessment for Pesticides in Europe: Implementation \& Research Needs. A Report from the European Workshop on Probabilistic Risk Assessment (EUPRA) for the Environmental Impacts of Plant Protection Products, the Netherlands. Central Science Laboratory Publishers, Sand Hutton, York (2001) (http://fera.co.uk/ events/pastConferences/documents/eupraReport.pdf).

30. Johansson, I. and Lynöe, N. Medicine \& Philosophy: A Twenty-First Century Introduction. Heusenstamm: Ontos Verlag (2008).

31. Attorney General of the State of New York In the Matter of Monsanto Company, Respondent. Assurance of Discontinuance Pursuant to Executive Law §63(15). Attorney General of the State of New York, Consumer Frauds and Protection Bureau, Environmental Protection Bureau (1996).

32. Reregistration Eligibility Decision (RED). Glyphosate. EPA-738-R-93-014. Washington, DC: United States Environmental Protection Agency (1993).

33. FAO Specifications and evaluations for plant protection products: glyphosate N-(phosphonomethyl)glycine. 2000/ 2001. Food and Agriculture Organisation of the United Nations (2001) (http://www.fao.org/ag/AGP/AGPP/Pesticid/ Specs/docs/Pdf/new/glypho01.pdf). 